\title{
Du texte à l'intertexte. Le palimpseste Macron au révélateur de l'Intelligence artificielle
}

\author{
Damon Mayaffre*1, Camille Bouzereau**, Magali Guaresi***, Frédéric Precioso**** et \\ Laurent Vanni***** \\ *Université Côte d'Azur, CNRS, BCL (UMR 7320), France, e-mail: damon.mayaffre@univcotedazur.fr \\ **Université Côte d'Azur, CNRS, BCL (UMR 7320), France, e-mail: bouzereau.camille@gmail.com \\ *** Université Libre de Bruxelles, ReSIC, Belgique, e-mail : magali.guaresi@gmail.com \\ **** Université Côte d'Azur, CNRS, I32 (UMR 7221), France, e-mail frederic.precioso@univcoted'azur.fr \\ *****Université Côte d'Azur, CNRS, BCL (UMR 7320), France, e-mail: laurent.vanni@univcotedazur.fr
}

Résumé. L'intertexte est la condition de l'interprétation de tout texte. Cependant, sa matérialisation, son explicitation, son implémentation ont toujours paru difficile pour la linguistique textuelle. Cette contribution propose une matérialisation de l'intertexte au sein de corpus réflexifs numériques, et convoque de manière originale l'intelligence artificielle (deep learning, modèle convolutionnel) et la logométrie pour explorer de manière systématique l'intertexte ainsi matérialisé. Pour cette étude, nous postulons que le corpus élyséen depuis 1958 constitue l'intertexte des discours d'Emmanuel Macron, dans lequel le président puise de manière consciente ou inavouée pour construire ses discours. A titre d'exemple, nous mettons ainsi au jour automatiquement les empreintes grammaticales de Giscard dans le discours de Macron, et les emprunts lexicaux que le nouveau président concède à de Gaulle.

\begin{abstract}
Intertext is the condition for the interpretation of any text. However, its materialization, its explicitation, its implementation have always seemed difficult for textual linguistics. This contribution proposes a materialization of the intertext within "numerical reflexive corpora", and calls in an original way artificial intelligence (deep learning, convolutional model) and logometry to systematically explore the intertext thus materialized. For this study, we postulate that the Elysian corpus since 1958 constitutes the intertext of Emmanuel Macron's speeches, from which the president draws consciously or unconsciously to construct his speeches. As an example, we thus automatically bring to light the grammatical imprints of Giscard in Macron's speech, and the lexical borrowings that the new president concedes to de Gaulle.
\end{abstract}

\footnotetext{
${ }^{1}$ mayaffre@unice.fr
} 


\section{Introduction}

Les notions d'intertexte et d'intertextualité sont centrales dans la linguistique des textes depuis 50 ans, mais elles restent encore aujourd'hui nébuleuses. Personne en effet ne conteste ni leur pertinence ni leur fécondité, mais nul n'a réussi à les formaliser.

La définition d'intertexte consacrée en 1973 dans l'Encyclopaedia universalis par Roland Barthes, sous l'entrée " Texte (théorie du) », dit tout de la force du concept en même temps que de sa fragilité scientifique :

Tout texte est un intertexte ; d'autres textes sont présents en lui, à des niveaux variables, sous des formes plus ou moins reconnaissables [...] ; tout texte est un tissu nouveau de citations révolues [...]. L'intertexte est un champ général de formules anonymes, dont l'origine est rarement repérable, de citations inconscientes ou automatiques, données sans guillemets. [Encyclopaedia universalis, ed. 1995, Tome 22, p. 372]

Admettons donc depuis Lotman, Kristeva, Barthes, Genette, Rifatterre, etc. sinon depuis Bakhtine ou Voloshinov, que l'intertextualité est la "condition de tout texte » (ibid.). En utilisant les mots des autres et de tous, le locuteur articule un discours qui n'est jamais totalement un inédit. Quand on parle, l'héritage en langue et en discours ne laisse qu'exceptionnellement la place à la néologie, et debout sur 2000 ou 3000 ans de culture textuelle, l'auteur est condamné à être un contrefacteur. Sans évoquer le cas caricatural et réducteur du plagiat, les écrits riches et nombreux sur l'intertexte, sur le dialogisme, la polyphonie ou l'hétérogénéité ${ }^{2}$ ont définitivement démontré qu'un texte n'est pas une monade ou un isolat mais un dialogue ou un carrefour, fait d'emprunts ou de reprises, de réponses ou de questions à l'endroit d'autres textes passés ou à venir, avec qui il communi(qu)e ; et sans lesquels il devient infra-interprétable.

Ajoutons encore, dans la posture herméneutique rastirienne qui est la nôtre, que lire c'est interpréter, et qu'interpréter c'est d'abord contextualiser, relier, mettre en correspondance, observer ce qu'il y a « entre ». Et cette contextualisation fondamentale se fait au plus haut niveau en versant le texte dans un déjà-dit, un autrement-dit ou un bientôt-dit, c'est-à-dire dans un certain intertexte. Dans cette perspective, nous considérons l'intertexte comme la condition de l'interprétation de tout texte.

Concrètement, la contextualisation pratique d'un texte à interpréter, dans une analyse, se fait à l'intérieur d'un certain corpus, défini de manière réflexive comme la matérialisation du contexte nécessaire à l'interprétation des textes qui le compose [cf. notamment Mayaffre 2002 et Rastier 2004]. Dit autrement, les corpus réflexifs que nous avons proposés ailleurs [Mayaffre 2002 et 2007] deviennent pour cette contribution l'intertexte objectivable des textes qui le composent. Précisons bien, puisque l'intertexte est potentiellement infini : le corpus réflexif constitue une forme explicitée ou objectivée d'un certain intertexte choisi pour l'analyse.

Cela étant dit, même réduit et matérialisé dans des corpus réflexifs dûment constitués, l'intertexte reste un concept mou sinon impuissant. Sa valeur élastique semble décourager la formalisation et interdire l'implémentation informatique : «à des niveaux variables » nous dit Barthes dans l'Encyclopaedia ; « formes plus ou moins reconnaissables » imprécise-t-il ;

\footnotetext{
2 Depuis la thèse de J. Kristeva en 1967, la littérature sur l'intertexte et les notions associées est vaste et passionnante ; parfois des ouvrages entiers. Nous donnons ici arbitrairement seulement deux références : le numéro spécial des Cahiers de praxématique, "Sémantique de l'intertexte », n³3, 1999 ; et l'ouvrage collectif de J. Bres et al. (dir.), Dialogisme et polyphonie - Approches linguistiques, Editions De Boeck-Duculot, 2005.
} 
« rarement repérable » sous-renchérit-il, « citations révolues », « inconscientes » et « sans guillemet ».

Nous souhaitons pourtant proposer ici un usage particulier de l'Intelligence artificielle des textes (deep learning, modèle convolutionnel (CNN)) susceptible d'envisager l'impossible, c'est-à-dire d'objectiver cet intertexte fuyant, mou ou liquide ; l'espoir est que le réseau de neurones artificiels relie, au sein du corpus, le texte-cible analysé (ici le texte de Macron dans son exercice de président entre 2017 et 2020) à son intertexte c'est-à-dire les textes qui le traversent (ici les discours présidentiels depuis 1958, soit ceux des prédécesseurs de Macron à l'Elysée).

Nous postulons en effet que l'intertexte - un des intertextes possibles - du discours de Macron est composé des discours que de Gaulle, Pompidou, Giscard, Mitterrand, Chirac, Sarkozy et Hollande ont pu prononcer avant lui, et dont le nouveau président s'inspire selon la contrainte générique (discours officiel présidentiel), la contrainte chronologique (le temps de la 5ème République) et la contrainte politique (discours de synthèse, " en même temps » de gauche et droite, en même temps gaulliste, giscardien, sarkozyste ou hollandais).

\subsection{Proposition méthodologique : un usage original du deep learning}

L'efficacité pratique des tâches d'apprentissage, puis de classification des textes par le machine learning ou le deep learning ne sont plus contestables aujourd'hui [par exemple Ducoffe et al. 2016 ou Brunet et Vanni 2019]. Depuis plusieurs années désormais, elle se chiffre par des taux d'exactitude (accuracy rate) spectaculaires.

3.1.1. Notre algorithme, implémenté dans le logiciel Hyperbase, a ainsi appris (learning) à reconnaître les discours de de Gaulle (phrases longues, plutôt nominales ou adjectivales, avec la «France » ou « l'État » comme premiers noms) comme ceux de Mitterrand (phrases courtes, plutôt verbales, avec le « je » et le « moi » comme centre d'intérêt personnel et «l'Europe » comme horizon). Elle a appris à reconnaitre les discours de Sarkozy (un lexique fort et une syntaxe faible) et ceux de Hollande (une syntaxe compliquée et un lexique affadi). Elle a appris à reconnaître les textes de Giscard (phraséologie technocratique ou didactique), ceux de Chirac (pleins de vide) ou ceux de Pompidou (style littéraire, riche voire ampoulé) ; et le jeu de validation (corpus de test composé de discours présidentiels anonymisés) permet d'affirmer que la machine retrouve automatiquement audessus de $92 \%$ des fois le bon auteur des discours. Sur un jeu de textes littéraires composé de l'essentiel de la littérature française aux $18^{\text {ème }}, 19^{\text {ème }}$ et $20^{\text {ème }}$ siècles, des résultats supérieurs, proches de $100 \%$ ont même été établi par Etienne Brunet, Laurent Vanni et Ludovic Lebart dans une étude monumentale qui brasse 50 auteurs de Racine à Giono, de Hugo à Yourcenar, de Proust à Le Clézio [Brunet et Vanni 2019 et Brunet, Lebart et Vanni 2020-sous presse].

En apprenant à la machine le discours élyséen depuis 1958, nous avons donc construit un certain horizon d'attente, ou au contraire un certain point de départ, des discours de Macron : selon nous, le module deep learning d'Hyperbase a appris un certain intertexte potentiel, dans lequel Macron pourra emprunter ou s'inspirer pour construire ses propres discours; ou encore, un certain intertexte dans lequel l'auditeur et l'analyste pourra faire résonner les discours de Macron pour les com-prendre et les inter-préter.

3.1.2. Puis, nous versons les discours de Macron dans ce corpus élyséen de référence (ou intertexte élyséen), en demandant à l'intelligence artificielle de rapprocher chaque phrase ou chaque paragraphe de Macron d'un des présidents précédents ; non sans artificialité donc, nous forçons l'algorithme à attribuer (prediction) les paragraphes de Macron à un de ses 
prédécesseurs au motif de ressemblances linguistiques détectées. Ainsi si Macron devait s'écrier à la tribune "Je vous ai entendu ! ", la machine attribuera ce passage à de Gaulle en référence sans doute au « Je vous ai compris ! " d'Alger en 1958. Si Macron devait prononcer « vous n'avez pas le monopole des sentiments », ou peut-être «vous n'avez pas l'exclusivité des sentiments ", la machine attribuera le passage à Giscard d'Estaing en référence au débat télévisé avec Mitterrand en 1974 durant lequel le candidat de droite avait répliqué au candidat de gauche « vous n'avez pas le monopole du cœur ». Pour donner un premier résultat réel de notre travail, lorsque Macron déclare lors de ses vœux aux Français, le 31 décembre 2018, « on ne peut pas travailler moins et gagner plus », Hyperbase attribue automatiquement la phrase à Sarkozy en référence au « il faut travailler plus pour gagner plus » que le président de droite avait souvent répété durant son mandat.

3.1.3. Enfin, dernière étape décisive pour l'étude linguistique, l'algorithme de déconvolution présenté à la communauté internationale lors du 56th Annual Meeting of the Association for Computational Linguistics (ACL), mis au point par [Vanni et al. 2018] permet de décrire le corpus : il s'agit non seulement d'extraire les phrases de Macron attribuées à de Gaulle ou Pompidou, Sarkozy ou Hollande, mais de surligner les éléments linguistiques qui ont participé à la décision. Ici, la méthode d'intelligence artificielle qui sert de guide (convolution puis déconvolution, et indice de reconnaissance des unités saillantes (Text Deconvolution Saliency -TDS)) est doublée par les procédés logométriques traditionnels (l'historique calcul des spécificités et le calcul des cooccurrences), afin d'affermir les résultats de l'IA par la statistique textuelle. Dans tous les cas, que cela soit en matière de classification ou de description, précisons que l'analyse se fait à trois niveaux linguistiques : les formes graphiques, les lemmes et les étiquettes morphosyntaxiques. Tant est si bien que tel extrait de Macron pourra être rapproché du discours de Giscard par exemple à cause de sa nature fortement nominale (combinaisons marquées de noms, d'adjectifs et de déterminants) ; tel extrait de Macron sera rapproché de Pompidou pour la variété des lemmes utilisés (concentration de plusieurs lemmes rares et précieux) ; tel extrait sera rapproché de Hollande pour sa combinaison de mots graphiques significatifs comme «territoires » (au pluriel) ou « investissements » (également au pluriel).

\subsection{Résultats : un intertexte pluriel pour un discours patchwork}

L'intertexte macronien est varié : sur les 10.000 paragraphes du corpus Macron analysés ${ }^{3}$, les taux d'emprunt ou d'inspiration se hiérarchisent ainsi (tableau 1) :

\begin{tabular}{|c|c|c|}
\hline \multirow{6}{*}{ 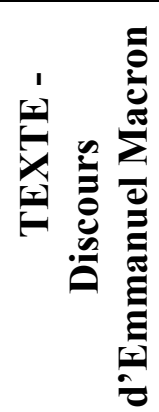 } & $\begin{array}{l}\text { INTERTEXTE - Présidents } \\
\text { inspirateurs }\end{array}$ & Taux d'inspiration \\
\hline & De Gaulle & $2 \%$ \\
\hline & Pompidou & $7 \%$ \\
\hline & Giscard & $3 \%$ \\
\hline & Mitterrand & $12 \%$ \\
\hline & Chirac & $21 \%$ \\
\hline
\end{tabular}

\footnotetext{
3 Précisons le corpus : composés de 1000 discours équivalent 3 millions de mots, le corpus recueille les principaux discours de de Gaulle, Pompidou, Giscard, Mitterrand, Chirac, Sarkozy, Hollande et Macron. Afin de donner une bonne représentativité au corpus, nous avons saisi les allocutions solennelles, les grands discours d'estrade, les interviews télévisées, les vœux ou les tribunes dans la presse.
} 


\begin{tabular}{|c|c|c|}
\hline \multirow{2}{*}{} & Sarkozy & $29 \%$ \\
\cline { 2 - 3 } & Hollande & $26 \%$ \\
\hline
\end{tabular}

Tableau 1. Intertexte de Macron ou sources d'inspiration de ses discours (en $\%$ de paragraphes)

L'intertexte de Macron répond donc grossièrement à la chronologie. Globalement, le discours de Macron emprunte davantage aux discours de ses prédécesseurs immédiats (Chirac, Sarkozy, Hollande) qu'à ceux de ses prédécesseurs plus lointains (de Gaulle, Pompidou, Giscard). Aux deux extrémités chronologiques notamment, Macron s'inspire seulement à hauteur de $2 \%$ de de Gaulle alors qu'il s'inspire à hauteur de $26 \%$ de Hollande. Néanmoins, d'intéressantes exceptions contredisent cette logique temporelle : Pompidou inspire plus Macron que Giscard, et Sarkozy - en tête du classement avec $29 \%$ - inspire plus le nouveau président que Hollande.

$\mathrm{Si}$ ce classement général est riche d'information, c'est l'examen linguistique systématique que nous avons fait des phrases attribuées à de Gaulle et Pompidou, Giscard et Mitterrand, Chirac, Sarkozy et Hollande qui est intéressant [Mayaffre 2020 - sous presse] : il en dit long sur le discours patchwork d'Emmanuel Macron qui diversifie son intertexte et multiplie les sources pour incarner une forme de syncrétisme discursif susceptible peut-être de réconcilier les mémoires et de résonner favorablement chez plusieurs types de citoyens.

Nous pourrions ainsi nous attarder sur les intertextes les plus évidents (ceux par ordre hiérarchique qui reprennent du Sarkozy et du Hollande) mais choisissons ici deux intertextes plus ténus - tenus mais effectifs - dans le discours : l'intertexte qui renvoie à de Gaulle et celui qui renvoie à Giscard. Le premier est surtout lexical, le second surtout grammatical.

\subsubsection{L'intertexte lexical gaullien chez Macron}

Ce qui reste de la rhétorique gaullienne dans le corpus Macron est évanescent ; évanescent et pourtant essentiel. L'ordinateur ne repère qu'une petite minorité de phrases affiliées à de Gaulle ( $2 \%$; tableau 1$)$, mais ces phrases peuvent marquer les esprits.

Les passages d'inspiration gaullienne ou intertexte gaullien que le deep learning met en exergue dans le corpus Macron concernent presque toujours la geste régalienne, qui célèbre la force et l'unité, la souveraineté et la grandeur politique. Un premier extrait dans lequel l'algorithme souligne les zones d'activation du réseau de neurones artificiels (Text Deconvolution Saliency (TDS)) dit l'essentiel :

Aussi, à côté de ces six batailles pour la souveraineté, c'est la bataille pour l'unité que je veux conduire. Nous n'aurons pas d'Europe forte et souveraine si elle n'est pas unie, tenue en elle-même, cohérente. Perdre cette unité c'est prendre le risque de revenir à nos déchirements mortifères et à l'hégémonie destructrice. ${ }^{4}$

De fait, dans ce passage repéré automatiquement dans le corpus de Macron, tout renvoie au discours de de Gaulle. Le terme «bataille », aujourd'hui pour partie suranné mais ici deux fois répété, donne une allure militaire au propos du jeune président, qui n'est pas sans rappeler l'uniforme du vieux général ; et le verbe « conduire » (conduire la bataille) rappelle

\footnotetext{
${ }^{4}$ Macron, 26 septembre 2017, discours sur l'Europe à la Sorbonne. Rappelons : les mots soulignés ont été activés par le réseau de neurones artificiels pour reconnaître ce passage de Macron comme ressemblant au discours de de Gaulle ; pour nous il s'agit des saillances de l'intertexte gaullien dans le discours de Macron.
} 
le leader historique sinon le guide ou le condottiere $e^{5}$. Les « déchirements mortifères » ou "l'hégémonie destructrice » ressemblent à des grandiloquences du discours politique, dont de Gaulle avait lui-même hérité de la rhétorique de la $3^{\mathrm{e}}$ République. Enfin et surtout, la question de «l'unité », directement reliée à celle de la souveraineté populaire (« souveraine ») renvoie à l'essence du gaullisme de 1958 et du gaullisme de toujours : un peuple uni derrière son chef légitime.

Cependant, dans cet extrait comme ailleurs dans le corpus Macron, la force novatrice du discours est d'utiliser cette dimension régalienne, haute en couleur, pour célébrer non pas la France, comme de Gaulle avait pu le faire, mais l'Europe. Le patriotisme du $\mathrm{XXI}^{\mathrm{e}}$ siècle n'est plus celui du $X^{\mathrm{e}}$ siècle : il s'est déplacé sur un nouvel objet. Si Macron est un de Gaulle, il prétend être un de Gaulle européen. Autrement dit, le texte de Macron diffère de celui de de Gaulle quand bien même son intertexte résonne de mots utilisés par de Gaulle.

C'est, en effet, exactement le même phénomène que l'intelligence artificielle repère dans la phrase suivante prononcée par Macron devant le Congrès et que l'algorithme attribue à de Gaulle :

Elle est affaiblie par les divisions, par le doute qui s'est installé dans notre peuple.

Pourtant, l'Europe est chez nous autant que nous sommes en Europe. Parce qu'il est impossible de penser notre destinée continentale autrement qu'au travers du projet européen. ${ }^{6}$

L'emphase lexicale gaullienne est ici à nouveau évidente, et ici à nouveau appliquée à l'Europe. Dans ce passage de 2017, le «peuple » est convoqué comme en 1958. D'un point de vue statistique, le mot est une grande spécificité gaullienne que ressuscite pour partie Macron à soixante années de distance ; là où un Giscard, un Chirac, un Sarkozy ou un Hollande, l'ont pour partie oublié (figure 1).

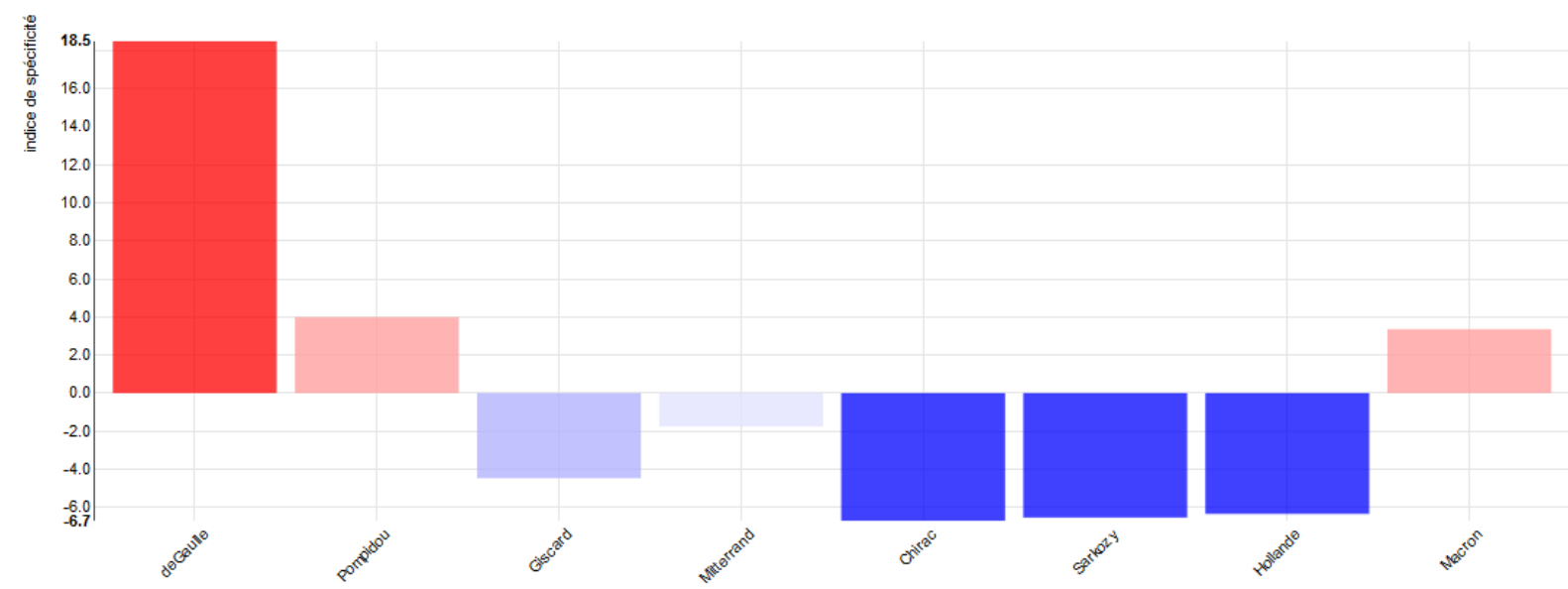

Figure 1. "Peuple » dans le corpus présidentiel, sur-utilisé par de Gaulle (et Macron) (HYPERBASE 2020).

Comme en 1958, Macron ne glose pas sur l'anecdote ou la simple actualité mais plus hautement sur la « destinée » ou encore le « destin », mots aussi typiquement gaulliens, emplis d'histoire et de sacralité, que le nouveau président sait également réutiliser (figure 2).

\footnotetext{
5 Par exemple : «L'appel qui m'est adressé par le pays exprime son instinct de salut. S'il me charge de le conduire, c'est parce qu'il veut aller, non certes à la facilité, mais à l'effort et au renouveau » (de Gaulle, 28 décembre 1958).

${ }^{6}$ Macron, 3 juillet 2017, discours devant le Congrès à Versailles.
} 


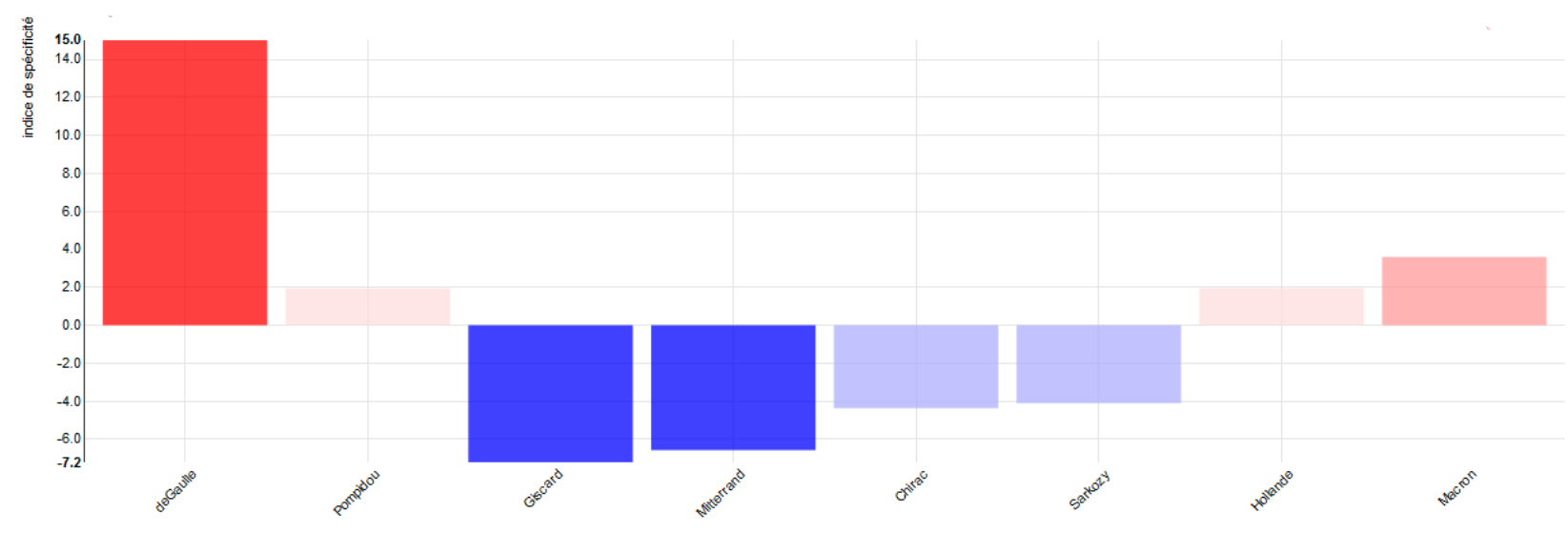
2020)

Figure 2. «Destin » dans le corpus présidentiel, sur-utilisé par de Gaulle et Macron (HYPERBASE -

Comme en 1958 surtout, c'est la « division » qui est le maître mot pour désigner le maître mal. A l'image de " peuple » ou de " destin ", " division » est spécifique des deux hommes (respectivement +3 et +4 ) et, sans nuance, de Gaulle s'en prend en 1962 au " démon des divisions » avant que Macron, lui, ne s'en prenne en 2018 au «poison de la division » ${ }^{7}$. Après les événements de 1968, de Gaulle espérait :

... puissions-nous, en dépit des heurts, des secousses, des divisions, qui ont encore une fois failli briser notre pays, nous retrouver tous, nous respecter mutuellement, nous rapprocher les uns des autres, dans notre unité nationale...

Et Macron de formuler cet espoir pour 2020 après les événements des « gilets jaunes » et les manifestations contre la réforme des retraites :

Enfin, 2020 doit ouvrir la décennie de l'unité retrouvée de la Nation. Je vois trop de divisions au nom des origines, des religions, des intérêts. Je lutterai avec détermination contre les forces qui minent l'unité nationale... ${ }^{9}$

Pour comprendre ces voix entremêlées du gaullisme et du macronisme, ces empreintes de Gaulle sur le palimpseste Macron ou ces emprunts que le nouvel impétrant concède au fondateur de la $5^{\mathrm{e}}$ République, rappelons-nous par exemple les phrases introductives de la première allocution du général adressée aux Français, lorsqu'il revient aux affaires en 1958 ; d'évidence Emmanuel Macron les a lues avant de prononcer certaines de ses envolées à la tonalité identique :

L'unité française se brisait. La guerre civile allait commencer. Aux yeux du monde, la France paraissait sur le point de se dissoudre. C'est alors que j'ai assumé la charge de gouverner notre pays. [...] Car, depuis 12 ans, le régime des partis, flottant sur un peuple profondément divisé au milieu d'un univers terriblement dangereux, se montrait hors d'état d'assurer la conduite des affaires. ${ }^{10}$

$\mathrm{Ou}$, ce passage après les événements de mai 1968 qui firent chanceler un instant le pouvoir :

\footnotetext{
${ }^{7}$ Respectivement : de Gaulle, 20 septembre 1962, allocution télévisée ; Macron, 16 octobre 2018, allocution télévisée.

${ }^{8}$ De Gaulle, 29 juin 1968, allocution radiotélévisée.

${ }^{9}$ Macron, 31 décembre 2019, vœux aux Français.

${ }^{10}$ De Gaulle, 13 juin 1958, allocution radiotélévisée
} 
Françaises, Français, voilà le chemin qu'il faut suivre et que ma vocation et mon mandat me commandent de vous montrer puisque le destin est en jeu. Mais quand demain, comme je l'espère, vous en aurez ainsi démocratiquement décidé, alors puissions-nous, en dépit des heurts, des secousses, des divisions, qui ont encore une fois failli briser notre pays, nous retrouver tous, nous respecter mutuellement, nous rapprocher les uns des autres, dans notre unité nationale! !l

De fait, Macron, un demi-siècle après de Gaulle, n'hésite pas à décrire lui-aussi l'état de la France comme une nation en perdition à cause de ses déchirements, ses rancœurs, ses fractures; et il le fait avant les événements des « gilets jaunes » qui durant l'hiver 2018-2019 interrogent objectivement l'état politique, social ou psychologique du pays.

Dans ces moments que nous vivons, faits de division parfois profonde, de doutes, de drames, mais aussi d'ambition indispensable pour que notre pays puisse redevenir conquérant dans ce siècle qui s'ouvre, j'ai besoin de vous ! ${ }^{12}$

Ainsi, en 2017, comme chez de Gaulle auparavant, l'appel au peuple («j'ai besoin de vous ») permet, dans un lexique dramatique (" conquérant ", " siècle ", " drames », « doute »), de remédier au mal de la « division » qui rongerait la société.

A propos de la difficile question du terrorisme, Macron reprend cette tonalité de discours avec des mots plus forts encore puisque les affres de la "guerre civile » sont évoquées, comme elles étaient évoquées par de Gaulle :

Il me suffit de constater que les terroristes veulent éroder les fondations de notre pays et de notre République pour provoquer un effondrement moral et déclencher une guerre civile. $^{13}$

De Gaulle a usé et abusé de cette rhétorique catastrophiste d'où découle « l'appel au peuple »; rhétorique que l'on a pu résumer historiquement dans la formule " moi ou le chaos ». Il le fit pour justifier son opposition à Vichy, sa démission en 1946, son retour en 1958, son élection en 1965 ou son maintien au pouvoir en 1968. Il fut même obligé de s'en expliquer publiquement à la télévision pour convaincre les Français de voter pour son nom lors de la première campagne présidentielle. Et sa dénégation dans un style impersonnel olympien, loin de convaincre, accable sa modestie :

Comme vous l'avez remarqué, je n'ai pas dit "moi", et je n'ai pas dit "le chaos". J'ai dit, et je répète ceci, s'il devait arriver le 19 décembre que le peuple français décidât d'écarter de Gaulle, c'est-à-dire, tranchons le mot, de renier ce qui est une partie de son histoire, et, je le crois, excusez-moi, encore aujourd'hui pour le moment, une nécessité nationale; si le peuple français en décidait ainsi, je suis convaincu que le régime des partis revenant..., ce serait pour le pays un immense malheur. ${ }^{14}$

Dans la bouche d'Emmanuel Macron, alors qu'on est loin de la Résistance ou de la guerre d'Algérie, l'évocation d'une France ravagée par ses factions ou ses divisions, quasi au

\footnotetext{
${ }^{11}$ De Gaulle, 29 juin 1968, allocution radiotélévisée.

12 Macron, 23 novembre 2017, discours aux maires de France.

13 Macron, 31 août 2017, interview dans Le Point. D'autres occurrences de «guerre civile » existent dans le corpus Macron, comme lors de son allocution télévisée du 14 novembre 2018, ou à propos de l'Europe : «Et donc au lieu de concentrer toute notre énergie sur nos divisions internes, comme nous le faisons maintenant depuis trop longtemps, au lieu de perdre nos débats dans une guerre civile européenne... » (Macron, 26 septembre 2017, discours sur 1'Europe).

${ }^{14}$ De Gaulle, 16 décembre 1965, interview radiotélévisée. Sur le mot « chaos », en juillet 2017, le chroniqueur des « Matins de France Culture » reprend en titre de son billet ce «Moi ou le chaos » à propos de Macron. Et Macron dira à propos des débordements des «gilets jaunes » à Paris : "Les coupables de ces violences ne veulent pas de changement, ne veulent aucune amélioration. Ils veulent le chaos $»\left(1^{\text {er }}\right.$ décembre 2018 , discours Buenos Aires). Echos intertextuels?
} 
bord de l'affrontement, que seul l'homme providentiel incarnant l'unité du peuple peut sauver, paraîtra surjouée. Mais il parvient à tromper la machine, comme lors du discours de la victoire en mai 2017 que l'intelligence artificielle attribue à de Gaulle :

Nous ne céderons rien à la peur, nous ne céderons rien à la division, nous ne céderons rien au mensonge, nous ne céderons même rien à l'ironie, à l'entre-soi, à l'amour du déclin ou de la défaite [...]. Ce ne sera pas tous les jours facile, je le sais. La tâche sera dure. Je vous dirai à chaque fois la vérité. [...]. Je combattrai pour vous contre le mensonge, l'immobilisme, l'inefficacité, pour améliorer la vie de chacun. Je respecterai chacune et chacun dans ce qu'il pense et dans ce qu'il défend. Je rassemblerai et je réconcilierai car je veux l'unité de notre peuple et de notre pays. ${ }^{15}$

Dans un de ses discours les plus construits, celui du 9 juillet 2018 devant le Congrès réuni à Versailles, Macron prononce ainsi des phrases aux accents évidents et à la solennité identifiable. Les mots activés par les réseaux - et la combinaison de ces mots dans un même paragraphe - nous éclairent sur l'intertexte mobilisé :

Tel est, Mesdames et Messieurs, le cap que je fixe à la France, vous l'aurez compris, je souhaite renouer avec ce projet français que nous avons perdu de vue trop longtemps par frilosité ou par confort intellectuel. Il suppose, je l'accorde, de vouloir s'affranchir des querelles où nous nous sommes en quelque sorte confondus ou auxquelles nous nous sommes longtemps habitués. Ce projet ne peut se déployer que si nous en finissons avec ce renoncement où nous nous sommes enfermés depuis 40 ans qui voudrait que la France ne soit qu'une puissance moyenne. Cette idée nous a étouffés et meurtris. Je crois, moi, que la France a les moyens de devenir de nouveau une puissance du XXIine siècle. ${ }^{16}$

De Gaulle ne dénonçait pas « 40 ans » d'immobilisme mais, constamment, les 15 ans d'impuissance de la $4^{\mathrm{e}}$ République dont l'avènement gaulliste sonnait heureusement la fin. Mutatis mutandis, le souffle des mots est identique chez les deux hommes. Un vieux monde, de " divisions », de " querelles », de " partis » ou de " chaos », aux mœurs politiques dépassées, s'écroule, et une ère nouvelle s'ouvre sur les traces du chef charismatique qui incarne l'union et la grandeur de la nation.

Les discours de Macron frappent ainsi les esprits car ils reprennent l'épopée gaulliste avec sa dimension héroïque, prophétique ou tragique. Comme en pleine Guerre mondiale sur Radio-Londres, comme en pleine guerre d'Algérie sur la future ORTF, Macron n'hésite pas à saturer le tableau dans une solennelle intervention télévisée :

Nous vivons, mes chers compatriotes, une situation inédite, une époque nouvelle, et nous serons jugés à l'aune de notre lucidité et de notre courage. Nous ne sommes plus aux temps innocents où l'on parlait de la fin de l'histoire et du tragique et où l'horizon de la politique était en quelque sorte l'extension de la société de consommation. Non. Le monde se fracture. De nouveaux désordres apparaissent. Et l'Europe bascule presque partout vers les extrêmes et à nouveau cède aux nationalismes [...]. C'est pour cela que je demande au gouvernement d'agir en ayant conscience de ce moment, et de prendre pour ce faire des décisions vigoureuses afin que le pays conserve cette maîtrise de son destin. [...] Je crois, mes chers compatriotes, dans ce ressaisissement de notre nation. Je crois dans notre capacité à porter cette voix française qui est tant attendue en Europe et dans le monde. Je crois dans ce projet que je veux porter en Europe, c'est pour cela que je souhaite le rassemblement le plus large. ${ }^{17}$

\footnotetext{
${ }^{15}$ Macron, 7 mai 2017, discours de la victoire.

${ }^{16}$ Macron, 9 juillet 2018, discours devant le Congrès à Versailles.

${ }^{17}$ Macron, 16 octobre 2018, allocution télévisée.
} 
Ici, dans cette intervention télévisée, il s'agissait seulement de remplacer un ministre de l'Intérieur démissionnaire, Gérard Collomb, et de changer quelques secrétaires d'État ; et les mots, trop en relief, tombèrent à plat. Mais la dimension épique d'un discours qui mobilise «l'univers », le « destin », « l'avenir », «l'espoir », le « peuple » ou la « nation » donne au propos macronien un horizon historique ambitieux que le parler présidentiel avait pour partie perdu : c'est l'intertexte lexical gaullien, bien identifié par les citoyens français et par le deep learning, qui permet de véhiculer ce souffle.

\subsubsection{L'intertexte morpho-syntaxique giscardien chez Macron}

Dans le corpus présidentiel, Macron puise peu dans l'intertexte de Giscard d'Estaing (3\%, tableau 1), moitié moins qu'il puise dans celui de Pompidou par exemple. Et ce sont des emprunts peu flatteurs qu'Hyperbase finit par repérer à la marge du discours : un langage technocratique, notionnel, austère et austéritaire.

Tous les deux énarques, tous les deux inspecteurs des Finances, tous les deux ministres de l'Économie, Macron et Giscard utilisent en commun une langue technique et notionnelle qui fige et dépersonnalise le discours ; une forme de langue de bois a-politique qui pédagogise l'économie, la libre entreprise et l'équilibre des budgets ; une langue ou une « ENAlangue $»{ }^{18}$ qui désidéologise la prose présidentielle pour en faire un discours professoral conceptuel et " techno ", un cours magistral d'HEC ou de droit administratif, un mode d'emploi aseptisé sur l'économie de marché en temps de crise.

Par exemple, Macron explique la suppression des emplois-aidés en une phraséologie que la machine reconnait comme giscardienne :

C'est de la subvention déguisée vers les collectivités locales ou le secteur associatif.

Ces secteurs ont toute leur utilité, mais, dans ce cas, cela doit entrer dans la dotation des collectivités locales ou dans les subventions aux associations, mais qu'on ne nous dise pas que c'est une politique de l'emploi. Le taux de retour à l'emploi durable des personnes concernées est en effet très faible. ${ }^{19}$

La formule nominale et absconse " le taux de retour à l'emploi durable », les simples noms « collectivités », « secteur », « dotation » ou « subvention » sont caractéristiques de cette novlangue politico-économique des services administratifs de l'État ou du monde des grandes entreprises.

Et plus loin dans cette interview, Macron précise la mise en place des Ordonnances travail :

Mais la branche, c'est-à-dire les représentants des entreprises et des salariés qui exercent des métiers similaires ou travaillent dans les mêmes types d'activités, c'est un progrès dans la meilleure prise en compte de la réalité économique. Ensuite, les branches sont indispensables parce que beaucoup d'entreprises, mal outillées ou trop petites, ne peuvent ou ne souhaitent pas négocier des accords complexes. ${ }^{20}$

" Accord », « branche », « types d'activités », « prise en compte de la réalité économique », « représentants des entreprises » : le lexique et plus encore, comme nous allons le voir, la grammaire sont caractéristiques.

Cette technicité d'un langage désincarné atteint parfois des hauteurs chez Macron comme elle atteignait toujours des sommets chez Giscard. Par exemple, lorsque Macron parle

\footnotetext{
${ }^{18}$ Christian Delporte, Une histoire de la langue de bois, Paris, Flammarion, 2009.

${ }^{19}$ Macron, 31 août 2017, interview dans Le Point.

${ }^{20}$ Ibid.
} 
de la santé, normalement vecteur d'émotion et de pathos, il s'enferme dans une langue morte qu'il semble reprendre à son prédécesseur :

Je souhaite que nous puissions créer, sur cette base, un véritable hub des données de santé, structure partenariale entre producteurs et utilisateurs des données, qui pilotera l'enrichissement continu mais aussi la valorisation du système national de données de santé, pour y inclure, à terme, l'ensemble des données remboursées par l'Assurancemaladie, en ajoutant les données cliniques des hôpitaux, les données de la médecine de ville, ainsi que les données de grande qualité, scientifiques et médicales, créées dans le cadre de cohortes nationales ${ }^{21}$

Les mots utilisés sont ceux du XXI ime siècle bien sûr, et Giscard n'employait ni «hub de données », ni "structure partenariale entre producteurs et utilisateurs des données », mais cette succession de syntagmes nominaux et de notions techniques est, selon la machine, typiquement giscardienne.

Car, à l'analyse, c'est moins le lexique que la machine détecte que l'étiquette morphosyntaxique et l'enchainement syntaxique : l'intertexte giscardien dans le discours de Macron est avant tout grammatical.

En effet « la valorisation du système national de données de santé », qui se schématise grammaticalement en " déterminant/nom + déterminant/nom/adjectif + déterminant/nom + déterminant/nom » est typique. La langue du septennat Giscard a marqué l'apogée de cette phraséologie nominale où les compléments du nom se succèdent en cascade pour nommer des réalités ou des processus techniques de plus en plus compliqués : Giscard a, dans le corpus élyséen (1958-2020), le taux de noms, de l'enchaînement "déterminant + nom » ou de l'enchaînement « déterminant + nom + préposition + nom » (par exemple chez Macron : « un système de santé », " la possibilité de règlement ») le plus spectaculaire de la $5^{\mathrm{e}}$ République (figure 3).

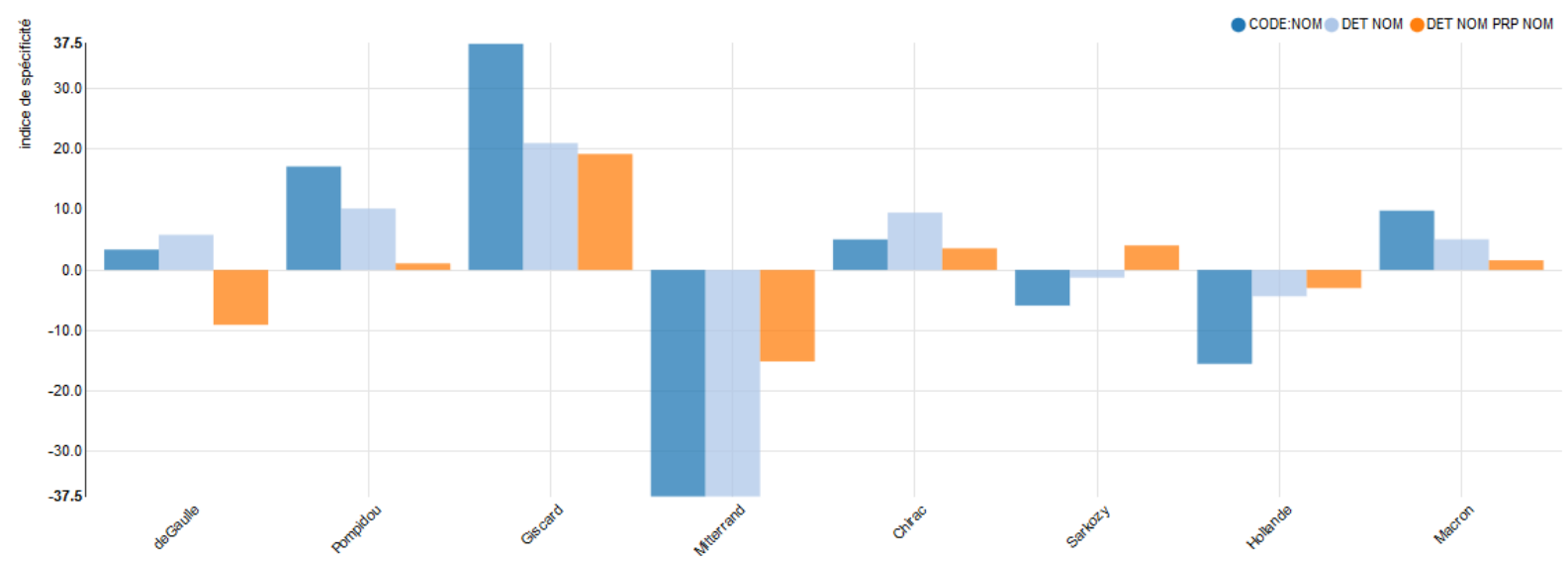

Figure 3. Sur-utilisation des noms et du discours nominal dans le corpus (HYPERBASE - 2020)

Cette rhétorique du nom, du complément du nom, du syntagme nominal est seulement légèrement fréquente chez Macron, mais lorsqu'il emploie « la réforme du marché du travail », « les acteurs du monde du logement », " les règles de recours au CDD », « la réalité des situations des collectivités territoriales », « la loi d'orientation des mobilités », etc., il semble comme Giscard en son temps, vouloir resserrer la chose publique dans un jargon technicotechnique et figer le débat dans des mécanismes naturalisés. Il euphémise la confrontation politique pour réduire le discours à la manipulation de notions et de sigles dont le but semble

\footnotetext{
${ }^{21}$ Macron, 29 mars 2018, discours AI for Humanity au Collège de France.
} 
de réifier les rapports sociaux, comme avait voulu le faire avant lui le président qui entendait rassembler, au centre, 2 Français sur 3.

$\mathrm{Du}$ reste, la contextualisation des structures nominales mentionnées ci-dessus est instructive sur cette ENAlangue. La formule nominale issue du débat sur la Loi travail durant l'été 2017, « les règles de recours au CDD », mérite d'être précisée pour que le citoyen puisse la comprendre et en débattre. Mais Macron indique seulement « les règles de recours au CDD peuvent se faire au niveau de la branche $»^{22}$ ajoutant ainsi une notion technique supplémentaire (la «branche ») à un propos qui l'était déjà doublement (" les règles de recours » et le « $\mathrm{CDD} »)$. De la même manière, à propos de « la réforme du marché du travail », il croit bon d'ajouter : "La réforme du marché du travail est une réforme de transformation profonde » ${ }^{23}$ ajoutant ici une notion abstraite («transformation ») à des concepts fuyants ou complexes (« réforme » ou « marché du travail »).

En l'occurrence, l'opposition de gauche et les syndicats dénoncent immédiatement, derrière ces deux derniers exemples (« règle de recours CDD », « réforme du marché du travail »), des Ordonnances travail qui, selon eux, réduisaient fondamentalement les droits des travailleurs et constituaient une des régressions sociales les plus importantes depuis l'aprèsguerre. Mais les phrases citées offrent objectivement peu de prise sémantique, politique ou idéologique à l'adversaire, au regard, soit de leur vacuité, soit de leur technicité ; et les Ordonnances de 2017 s'appliqueront sans beaucoup de contestation dans le pays.

Les exemples de cet intertexte grammatical giscardien pourraient être multipliés, comme cet extrait caricatural, issu d'un discours qui, ici, loin de réussir à neutraliser les oppositions politiques, participera au contraire au malaise des " gilets jaunes » à l'automne 2018:

La loi d'orientation des mobilités, présentée hier en Conseil des ministres après une intense phase de concertations, propose une partie de ces solutions qui seront à construire sur le terrain. ${ }^{24}$

Ce type de discours fortement nominalisé autour de termes creux ou techniques de l'économie, souvent également autour de sigles plus ou moins opaques (TPE, APB, GPA, REP+, CSG, AMF, BTP, ALUR, APL, UNEDIC, IFI, etc.) a pu être décrit par Christian Delporte comme l'excellence de la langue de bois des décideurs, et comme une rhétorique de l'esquive d'hommes politiques soucieux de faire passer des réformes impopulaires par des discours aseptisés. Ce type de discours renvoie en effet toujours aux politiques déjà en place ( " loi d'orientation », " règles de recours », " réforme du marché du travail ») et à la doxa dominante, c'est-à-dire en l'occurrence au modèle économique en vigueur qui cherche à neutraliser les différends politiques, ou la lutte des classes, derrière des notions administratives, institutionnelles ou naturelles qui semblent s'imposer d'elles-mêmes (Guilbert 2011). Ce discours permet en fait de techniciser un débat, audible seulement par une élite initiée, pour ne pas avoir à discuter avec les citoyens ou les travailleurs des principes et des fondements. Il permet de se soustraire aux jugements axiologiques ou idéologiques du mal et du bien, du juste et de l'injuste, du vrai et du faux, et d'éviter la contradiction politique derrière un allant de soi qui tire sa force de la langue. C'est un discours " notionnel » au sens étymologique c'est-à-dire un discours de la connaissance, du savoir ou encore de l'expert ${ }^{25}$ :

\footnotetext{
${ }^{22}$ Macron, 31 août 2017, interview dans Le Point.

23 ibid.

${ }^{24}$ Macron, 27 novembre 2018, discours sur la transition écologique.

25 « Notion» est lointainement issu, via le grec et le latin, de l'indo-européen *gno qui signifie « savoir », et que l'on retrouve par exemple en anglais dans le verbe to know ; une notion est un savoir élémentaire et les discours notionnels sont tenus par les gens qui savent (ici Macron et Giscard d'Estaing).
} 
une rhétorique du « je sais pour vous » conclut Christian Delporte ${ }^{26}$. Et, de fait, Macron surutilise massivement le verbe " savoir » à la première personne (" je sais »), comme Giscard sur-utilise le verbe professoral « indiquer » : « je vous indique » (figure 4).

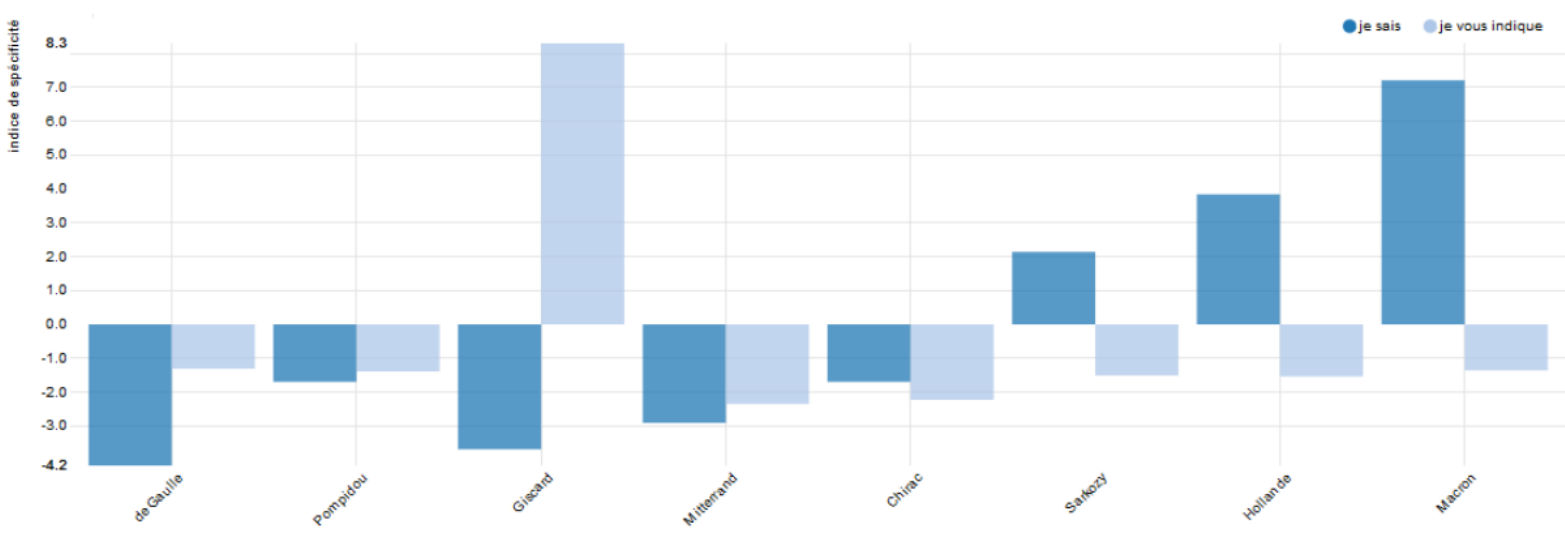

Figure 4. "je vous indique » chez Giscard ou « je sais » chez Macron (HYPERBASE - 2020)

Quand Macron imite Giscard, nous versons ainsi dans un discours sans visage ni patrie dont la conséquence est, au final, probablement contreproductive pour Macron comme elle le fut pour Giscard, en affaiblissant, par la forme, le message principal. En effet, le paradoxe de ce discours notionnel, descriptif, explicatif, stéréotypé est qu'il dégage une forme de fatalité la fatalité de choses déjà-là, la fatalité d'une pensée unique et d'un discours pré-construit, la fatalité d'une mécanique économico-administrative ou gestionnaire que l'on ne saurait changer -, là où la modernité revendiquée par les deux jeunes présidents réclamerait au contraire action, débat, volontarisme, et pourquoi pas polémique ? Ce « je sais pour vous », ou pire encore "le système sait pour vous ", " la langue parle à votre place " apparaissent comme un dessaisissement du politique au profit d'un ordre discursif (des formules figées) et d'un ordre économique (les politiques menées à l'échelle planétaire ou européenne depuis quelques décennies), c'est-à-dire comme un désinvestissement démocratique du président luimême qui semble utiliser un prêt-à-parler technique et convenu ; et les citoyens, à leur tour, d'être plongés dans l'impuissance politique. Sans doute est-ce pour cette raison que Macron emploie cet intertexte morpho-syntaxique giscardien (nom, complément du nom, complément du complément du nom) assez peu dans le corpus, pour rester fidèle au volontarisme horizontal et à l'élan démocratique vantés durant la campagne électorale 2017.

\section{Conclusion}

L'explicitation de l'intertexte est garant d'une certaine scientificité interprétative, dans la mesure où sa mise en place et sa communicabilité rendent une interprétation partageable. Il clarifie le terrain du face-à-face interprétatif en le rendant accessible aux regards extérieurs. En effet, une lecture qui expliciterait son parcours non seulement à travers les structures textuelles mais aussi les structures intertextuelles, offrirait une image claire, du moins une image moins fuyante de la compétence interprétative du lecteur et proposerait, en même temps, une grille d'appréciation, d'évaluation aussi de sa performance. ${ }^{27}$

\footnotetext{
${ }^{26}$ Chr. Delporte, Une histoire de la langue de bois, op. cit.. Sur le discours de l'expert, voir R. Cussó et C. Gobin dans un article au titre substantiel : «Du discours politique au discours expert. Le changement politique mis hors débat? », Mots, 88, 2008, p. 5-11.

${ }^{27}$ Ioannis Kanellos, « De la vie sociale du texte. L'intertexte comme facteur de la coopération interprétative », Cahiers de praxématique [En ligne], 33 | 1999, § 115.
} 
Nous partageons l'affirmation de I. Kannelos sur l'importance de l'intertexte et sur la nécessité d'essayer de l'expliciter pour permettre une interprétation critique. Et nous partageons son scepticisme fondamental quant à réussir à formaliser définitivement cet intertexte, puisque l'intertexte est discrétionnaire à chaque lecture, à chaque auteur, à chaque lecteur dans l'espace culturel infini des productions discursives et textuelles. Pour I. Kannelos, l'intertexte ne s'assimile à aucune "encyclopédie » aussi exhaustive soit-elle, l'intertexte «n'est pas le visage textuel du contexte » aussi large celui-ci puisse-t-il être, pas plus qu'il ne s'identifie à aucun « corpus ». ${ }^{28}$

Cependant, en renonçant à l'idéal pour le réalisable, nous avons proposé dans cette contribution un parcours méthodologique balisé dans un horizon interprétatif pragmatique et fini : nous définissons les grands corpus numériques réflexifs, précisément, comme une explicitation possible de l'intertexte - d'un certain intertexte comme il y a certains horizons d'attente - et convoquons le deep learning pour explorer - ou « apprendre » - cet intertexte.

Les développements récents de l'intelligence artificielle sur texte nous permettent ainsi de développer le programme exprimé par exemple par Glenn Roe qui, sans illusion pourtant sur les humanités numériques, invite à ouvrir une phase nouvelle des études littéraires :

...celle de la synthèse entre la textualité restreinte des collections numériques soignées et scientifiques et la contextualité grandissante des textes à l'ère de la numérisation massive. Nous entrons [...] dans le moment de l'intertexte; c'est-à-dire de l'interdépendance et de l'interconnexion des textes et des données... [C]ette intertextualité numérique requiert des approches synthétiques à l'étude littéraire, capables d'offrir en même temps, et la spécificité de l'œuvre d'art et la pluralité des systèmes d'information qui l'entoure ; méthodes qui facilitent, d'une manière transparente et intuitive... le mouvement entre la macroanalyse ou la lecture à distance des collections massives et la lecture intensive ou la microanalyse des textes numériques; mobilité bilatérale et réciproque, enfin, entre le texte et le contexte par l'intermédiaire de l'intertexte. En prenant l'intertextualité comme base théorique de nos recherches informatiques, nous nous plaçons consciemment dans la riche tradition de l'étude intertextuelle, telle qu'elle a été décrite par Julia Kristeva et Roland Barthes, ou bien de Harold Bloom, à la même époque. ${ }^{29}$

Concrètement, prolongeant les espoirs de Ludovic Tanguy et Théodore Thlivitis dans " Parcours interprétatifs (inter)textuels : vers une assistance informatique » [1999], nous demandons à l'algorithme de révéler les couches cachées ou profondes (deep) du texte qui semblent recéler ses empreintes inavouées et ses emprunts revendiqués : son intertexte.

Palimpseste. Révélateur numérique. Interprétation humaine.

\section{Bibliographie}

Barthes (R.) (1973), «Texte (théorie du) », Encyclopaedia universalis, ed. 1995, Tome 22, pp. 370-374.

Bres (J.) et al. (2005), Dialogisme, polyphonie : approches linguistiques, Paris, De Boeck.

Brunet (E.) et Vanni (L.) (2019), « Deep learning et authentification des textes », [En ligne], $\begin{array}{lllll}\text { Volume XXIV } & \text { - } & & 1 & \text { (2019). [http://www.revue- }\end{array}$ texto.net/docannexe/file/4194/texto_brunetvanni_deep_final.pdf. Consulté le 06/01/2020).

\footnotetext{
${ }^{28}$ Entre guillemets, les têtes de réflexion de I. Kannelos dans sa partie «2. Ce que l'intertexte n'est pas » (ibid.).

${ }^{29}$ Roe (G.) (2014), « L'étude littéraire à l'ère du numérique: du texte à l'intertexte dans les "digital humanities" », Philologie im Netz Beiheft, 7, p. 104.
} 
Brunet (E.), Lebart (L.) et Vanni (L.) (2020 - sous presse), «Littérature et intelligence artificielle » in D. Mayaffre et al. (sous la dir.), L'intelligence artificielle des textes. Points de vue critique, points de vue pratique, Paris, Champion, Lettres numériques.

Delporte (Chr.) (2009), Une histoire de la langue de bois, Paris, Flammarion.

Ducoffe (M.) et al. (2016), « Machine Learning under the light of Phraseology expertise: use case of presidential speeches, De Gaulle -Hollande (1958-2016) », in JADT 2016 - Statistical Analysis of Textual Data, Nice, Jun 2016, France, pp.157-168. [hal-01343209V2] ;

Kanellos (I) (1999), « De la vie sociale du texte. L'intertexte comme facteur de la coopération interprétative ", Cahiers de praxématique, 33, [En ligne http://journals.openedition.org/praxematique/1973 consulté le 06/01/2020].

Genette (G.) (1982), Palimpsestes, La littérature au second degré, Paris, Seuil.

Roe (G.) (2014), «L'étude littéraire à l'ère du numérique: du texte à l'intertexte dans les "digital humanities" », Philologie im Netz Beiheft, 7, 85-111.

Mayaffre (D.) (2002), "Les corpus réflexifs: entre architextualité et hypertextualité », Corpus, 1 [En ligne : http://journals.openedition.org/corpus/11, consulté le 02 janvier 2020].

Mayaffre (D.) (2007) «Philologie et/ou herméneutique numérique : nouveaux concepts pour de nouvelles pratiques ", in François Rastier et Michel Ballabriga (éds), Corpus en Lettres et Sciences sociales. Des documents numériques à l'interprétation, Toulouse, Put, 2007, pp. 1526. [hal-00551477].

Mayaffre (D.) (2020-sous presse), Macron par l'intelligence artificielle. Ses discours décryptés par la machine, La Tour-d'Aigues, Les éditions de l'Aube.

Rastier (F.) (2001), Arts et sciences du texte, Paris, Puf.

Rastier (F.) (2004), «Enjeux épistémologiques de la linguistique de corpus ». Texto ! [en ligne, Rubrique Dits et inédits. Disponible sur: http://www.revuetexto.net/Inedits/Rastier/Rastier_Enjeux.html - Consulté le 06 janvier 2020).

Rastier (F.) (2011), La mesure et le grain. Sémantique de corpus, Paris, Champion.

Rastier R. et Pincemin B (1999), «Des genres à l'intertexte », Cahiers de praxématique, 33 [En ligne : http://journals.openedition.org/praxematique/1974, consulté le 05 janvier 2020.]

Tanguy (L.) et Thlivitis (T.) (1999), "Parcours interprétatifs (inter)textuels: vers une assistance informatique ", Cahiers de praxématique, 33 [En ligne, http://journals.openedition.org/praxematique/1999 consulté le 04 janvier 2020].

Vanni et al. (2018), «Text Deconvolution Saliency (TDS) : a deep tool box for linguistic analysis », 56th Annual Meeting of the Association for Computational Linguistics, Jul 2018, Melbourne, France [hal-01804310]. 